\title{
Touching Precious Metals
}

\author{
Walo Wälchli
}

Schweizerische Edelmetallkontrolle, Basel, Switzerland

\begin{abstract}
The technique of touching as a means of assaying gold and other precious metal alloys has been extensively used by goldsmiths for several millenia. In this article the historical origins of touching are discussed and the process, and its tools and applications today are reviewed.
\end{abstract}

\begin{abstract}
Assay and hallmarking offices have the difficult task of assaying, accurately and preferably nondestructively, valuable jewellery articles which are often in finished form, polished, set with stones or pearls and which may even have undergone some treatment to improve the appearance or technical properties of their surfaces. Two methods are currently available for such assaying. The first involves the sampling by scraping for subsequent fire assay and the second is the touchstone test. Both methods have advantages and disadvantages, but a detailed discussion of their comparative merits falls outside the scope of this article.

Swiss assayers have a long tradition of successfully touching finished jewellery articles and have acquired by experience the ability to use the method at an exceptional level of accuracy. Thus, differences in precious metal contents as small as 10 to 20 parts per thousand may be established with confidence by the touchstone test. In testing against hallmarking standards, where negative deviations only are of concern, a better accuracy is normally required. Therefore, if the result of touching leaves the assayer in any doubt, he resorts to fire assay. It is not unusual for the result of the latter, very accurate method to be within 5 parts per thousand of that of the preliminary touchstone test!
\end{abstract}

\section{History}

Touching is among the oldest methods of assaying for noble metals. It was first referred to by the Greek poet and moralist Theognis of Megara (6th century B.C.) who made repeated symbolic mention of the 'gold testing stone' in his verse. A manuscript of the Arthaŝâstra, the 5th or 4th century B.C. treatises attributed to Kautilya, describes the use of touchstones in ancient India. Pliny the Elder (23 to 79 A.D.) recorded in his 'Natural History' that, using a touchstone to test ore,

'Persons of experience...can tell in a moment the proportion of gold there is in it, how much silver, or how much copper; and this to a scruple, their accuracy being so marvellous that they are never mistaken.' (Book XXXIII, chapter 43 ).
The Leyden Papyrus X, from Thebes, which was probably written early in the 4th century A.D., indicates that touchstones were employed by the ancient Egyptians.

From the 12th century onwards, goldsmiths' and silversmiths' guilds became established throughout Europe. The charters and rules of these associations make extensive mention of touching in connection with the control of official standards for gold- and silverware.

The introduction of printing, towards the end of the 15 th century, led to the publication of the first assay manuals which detailed the procedures of touching, and gave recipes of acid mixtures and descriptions of the touch-needles. However, it was only after the discovery of mineral acids by the alchemists that significant progress was made in touching and assaying. The use of nitric acid (first known as aqua fortis) and of aqua regia began in the first half of the 17 th century. Some 150 years later, proper test acid solutions were formulated and the first reference to them was made by the French chemist Vauquelin in 1799.

\section{Scope of the Method}

Touching is part of the art and science of assaying noble metals. Because of its simplicity, the method has found widespread application for testing noble metal articles of all types. It may be used with items of any shape, be they raw materials, semi-finished products or delicate pieces of jewellery. Touching does not cause significant damage, it requires a minimum of material and equipment, and may be performed very rapidly. In most cases, the method not only identifies qualitatively the constituents of a precious metal alloy, but also allows their quantitative determination. Touching is of particular interest when very little material is available for sampling and analysis - a typical test requires only about $0.5 \mathrm{mg}$ of alloy. Moreover, it is well suited to the testing of inexpensive articles, when the cost of fire assay cannot be justified, or of very valuable pieces, for which sampling by scraping is unacceptable. 
A gold alloy brooch and the accessories that are required to test it by touching

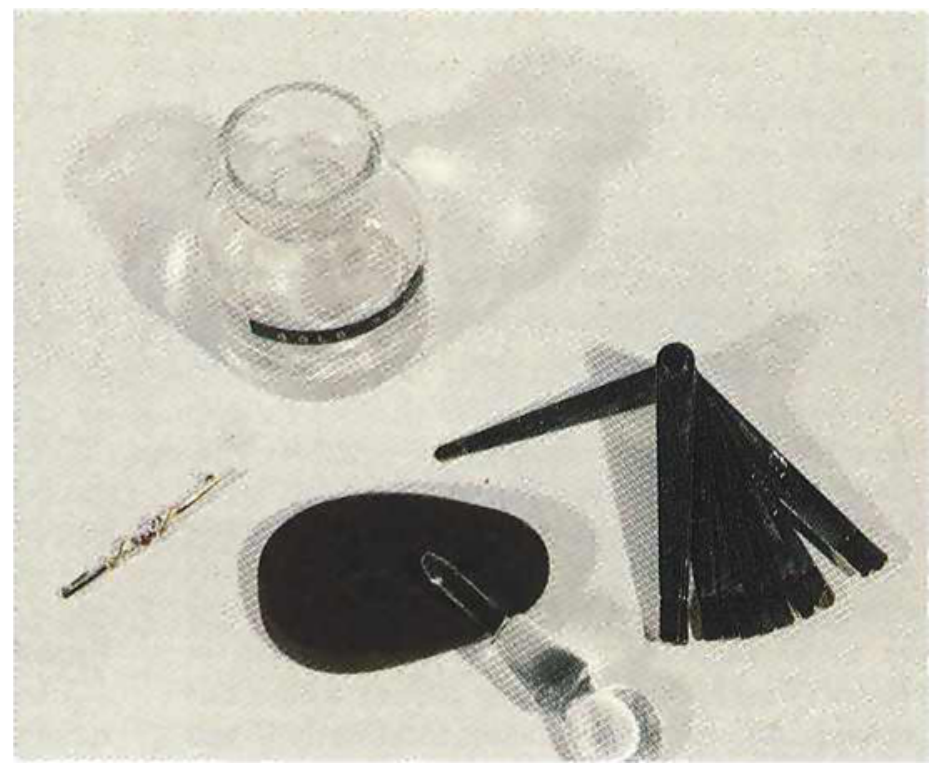

In summary, touching is used by the assayer to:

(1) Identify precious metals in alloys

(2) Identify many base metals in alloys

(3) Assay finished articles

(4) Test samples before using finer analytical methods

(5) Obtain some indication of the thickness of precious metal coatings. (This is done by producing from the sample a wavy linear rubbing of sufficient length to ensure wear through the coating. Then, the base metal portion of this trace is dissolved with an appropriate acid solution. The length of the remaining precious metal trace can finally be interpreted by the assayer. The method obviously requires much experience.)

\section{The Procedure}

Touching is essentially a comparative method which consists of the following operations:

First, the object made of the alloy to be tested is rubbed under firm and even pressure onto a hard, acid-resistant, lightly oiled touchstone until adjacent rubbings cover completely and uniformly an area 20 to $30 \mathrm{~mm}$ in length and 3 to $5 \mathrm{~mm}$ in width. Then, next to this, a similar film of metal is rubbed onto the stone from a needle made of a standard alloy of known fineness and matching colour. It is important that both the test and reference rubbings should be of similar sizes and intensities. Finally, the rubbings are wetted with an appropriate test acid solution. The acid preferentially attacks the base metals in the alloy, as well as silver. When sufficient time has elapsed for the action of the acid on the rubbings, these are wiped with filter paper. It is then possible to make a visual estimate of the fineness of the sample. Usually, the degree of attack, and therefore the fineness, are revealed by the coloration of the area of the rubbing which has been in contact with the acid: the lighter this is, the higher the fineness, and vice-versa. This is because, as a rule, alloys of low fineness are more readily and more completely dissolved than those which are rich in precious metals. Similar behaviour

and appearance of the test and reference rubbings are indicative of identical fineness.

\section{Accuracy}

Generally speaking, approximate results only can be expected from touching. Under optimum conditions, an experienced assayer can detect differences in fineness of 10 to 20 parts per thousand. Among the variables which affect accuracy are the quality of the stone, the type and range of needles at the disposal of the assayer, the strength of the acids he uses, his experience and the ambient lighting conditions. However, the most important factors are the properties and composition of the alloy tested. Very soft materials, such as fine gold or alloys containing more than 920 parts per thousand of gold, often do not leave rubbings on the stone, but 'smear'. Very hard alloys, such as some white golds, on the other hand, scratch the stone and are not amenable to touching.

\section{The Tools and Accessories}

It is apparent from the above that a touchstone, needles and acids are all the equipment that is required for touching.

\section{Touchstones}

In contrast to earlier times, when only black and dark grey stones were used, present practice employs both black and red touchstones.

The red stones, which are particularly well suited for touching gold, are called radiolarites, as they consist of radiolaria. These marine protozoa have siliceous skeletons which accumulate as radiolarian sludge which is a red, clayish, deep-sea sediment. Eventually time and pressure turn this sludge into the sedimentary rock radiolarite. 
The black stones consist of a siliceous slate which is a hard, dense and brittle mineral consisting predominantly of quartz and chalcedony. Its origin is similar to that of radiolarite, but in this instance the sedimentary sludge has been submitted to much higher pressure and possibly exposed to heat. Siliceous slate with a beautiful black colour imparted by carbonaceous constituents is called lydite.

A good quality touchstone is of uniform deep black or brownish red colour. It is free from veins or spots, fine-grained, acid-resistant and appropriately hard. The surface has to be ground to a matte finish and should not be polished, otherwise the rubbings would not adhere to the stone. Before use, the stone is lightly impregnated with a pure oil, as oil-free stones accept the metal rubbings only with difficulty and in patches. Touchstones are easily cleaned with a fine abrasive and can be used almost indefinitely if well cared for.

\section{Touch-Needles}

The alloys of known compositions, which are required by the assayer for reference, are commercially available as touch-needles or stars.

A needle consists of a brass or German silver shank to one end of which a piece of standard precious metal alloy has been soldered. The gold and silver contents of the standard alloy, and usually the details of other constituents in it, are inscribed on the shank.

Five- or six-pointed stars are made of base metal with lens-shaped pieces of standard precious metal alloys soldered to the tips of the branches.

The higher the accuracy demanded from touching, the larger the number of touch-needles which must be available to the assayer. When touching gold alloys, it is essential to have needles not only of various finenesses, but also of various colours for each standard caratage. Thus, wide assortments of needles of identical fineness and covering a range of colours are found in all assay offices.

\section{Acids}

The acid solutions used for touching gold, which should under no circumstance attack the rubbings too violently, consist either of diluted aqua regia or of nitric acid with copper chloride (Table I). These solutions are kept in bottles with stoppers suitably shaped to transfer the required amount of acid onto the rubbings. Assayers also use acid-resistant plastic dropping bottles.

\section{Touching Gold}

Touching may be used either to determine whether an alloy contains any gold at all or to determine the fineness of an alloy known to contain gold.

\section{Establishing the Presence of Gold}

After attack by diluted aqua regia or nitric acid, the rubbings left by an alloy of unknown composition appear as reddish brown or brownish black residues if gold is present in small quantities. These residues are dissolved in stronger aqua regia which is then absorbed onto filter paper. A drop of tin chloride solution is applied to the wet spot on the paper and the presence of gold is revealed by the development of a light to dark purple halo (Purple of Cassius).

Using a red, or especially a white, touchstone (Arkansas grinding stone, for example), this test is positive for gold concentrations in the alloy down to about 50 parts per thousand.

\section{Coloured Gold Alloys}

In order to determine the fineness of a coloured gold alloy of unknown caratage, a rubbing is tested with concentrated nitric acid, while a second one is tested with the acid solution for 18 carat alloys. If the first rubbing is attacked, then it may be concluded that the gold content of the alloy is below 500 parts per thousand. The situation is similar with the second rubbing, where attack indicates a gold content less than 750 to 800 parts per thousand.

Table I

Acid Solutions Used for Touching Gold Alloys

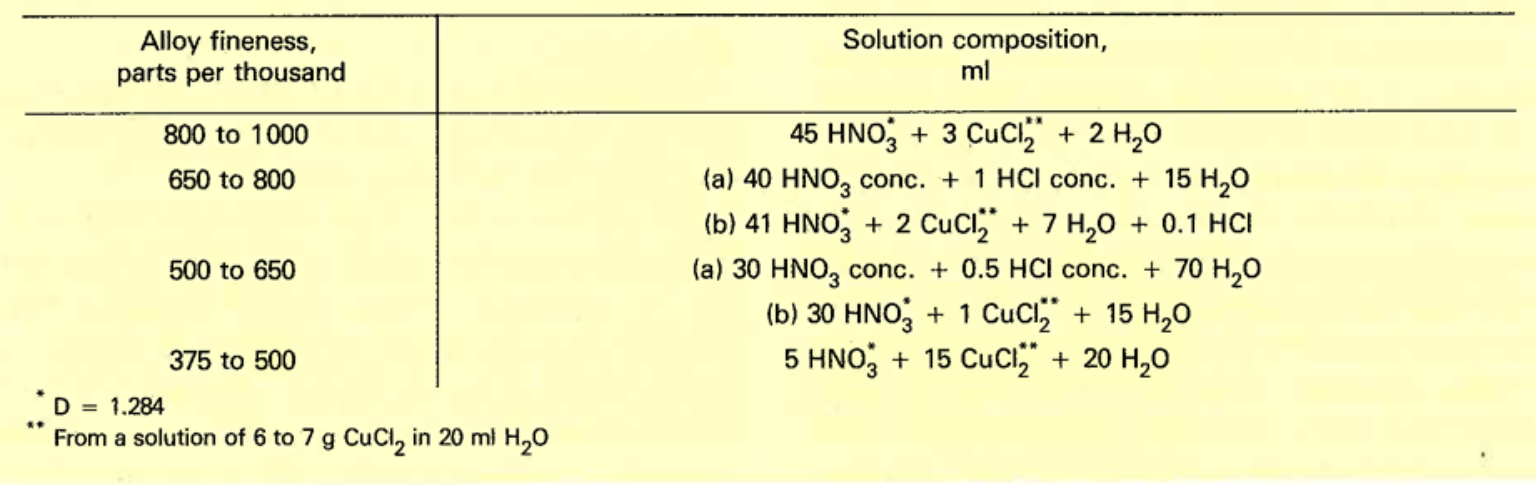



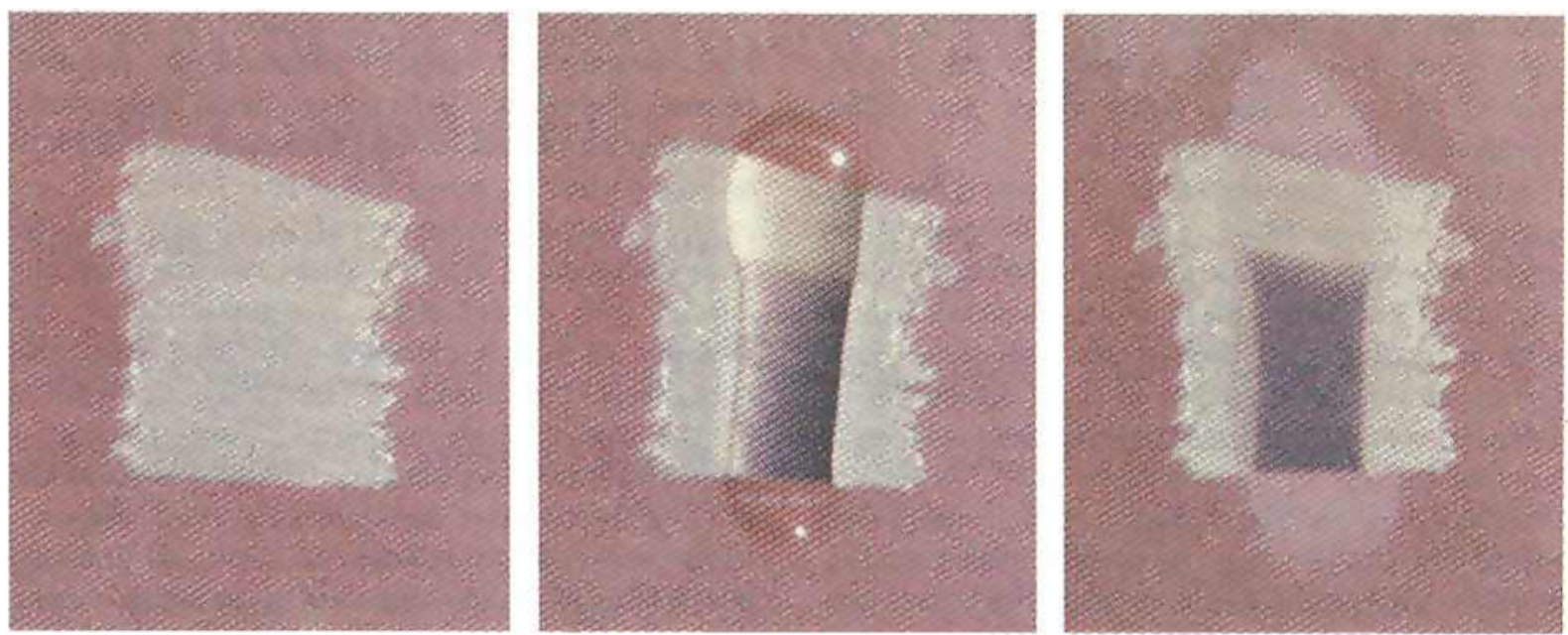

Testing of three side by side rubbings of gold alloys ( 585 parts per thousand at the bottom and in the centre, and 750 parts per thousand at the top)

Left: The rubbings as made from gold alloys

Centre: Attack by an acid solution

Right: After wiping the acid, the different behaviour of alloys of various finenesses is clearly visible

After this first approximate indication of the caratage of the alloy has been obtained, a more accurate determination of the fineness is made by comparing the behaviour of rubbings of the sample with that of rubbings of appropriate needles which should match closely the alloy to be identified not only in fineness, but also in colour. The process is pursued to the point where rubbings of the piece under test and of a particular needle behave in an identical manner when submitted to attack by the same acid solution. Apart from the fineness, the method also provides information on the approximate silver and copper contents of ternary gold-silvercopper alloys.

When touching for gold, it is important to bear the following in mind, in order to ensure maximum accuracy:

(1) If the action of the acid is excessively prolonged, the residues of the rubbings darken as a result of oxidation and the visual perception of small differences in fineness becomes difficult or even impossible

(2) It is often advantageous to place the stone under running water for the observation of the results of the attack

(3) Silver - present in most gold alloys - produces a milky precipitate of silver chloride, which turns blueish and is detrimental to accurate observations. As silver chloride is soluble in ammonia, it is recommended that the rubbings are treated with a solution of the latter after a first attack by the acid solution. Acid is then applied again for a short time before wiping with filter paper. This procedure assists in distinguishing small differences in fineness
(4) Upon removal of the acid, the residues of the rubbing tend to tarnish rapidly with time. This undesirable effect is particularly noticeable with copper-rich and zinc-containing gold alloys

(5) Attack on red gold rubbings is characterized by the evolution of gas. The attack is not complete and the excess acid should not be removed until after this evolution of gas has ceased

(6) Dilute gold alloys (those with a gold content below 400 parts per thousand) are advantageously tested on a red or white touchstone such as an Arkansas grinding stone, for instance. The test acid for these alloys is usually a dilute solution of nitric acid. A brown residue remains after removal of the acid, which is darker for alloys of very low gold contents. Finenesses down to about 100 parts of gold per thousand can be determined in this way.

\section{White Gold Alloys}

As a rule, it is not feasible to touch white gold alloys with the accuracy that is possible for their coloured counterparts. The main reason for this is that all white golds of fineness between 333 and 916 parts per thousand are of rather similar greyish white colour. Thus, it is not possible to derive any preliminary assessment of the gold content or the composition of these alloys from their colour, and the assayer has to rely entirely on his observations of the behaviour of the rubbings during attack by the acids in order to obtain such information. However, this behaviour is not dependent solely on the gold content, but is strongly influenced by the nature and relative amounts of other constituents in the alloy. This factor makes the selection of the appropriate test-needles very difficult 
and these play a much lesser role in touching white golds than yellow golds. Therefore, the results arrived at by touching white alloys may differ significantly from actual finenesses.

Nitric acid - concentrated or diluted - is normally used on rubbings of white golds of fineness below about 650 parts per thousand. The residues have a reddish colour after attack, which becomes lighter with increasing gold content and vice-versa. This feature allows the detection of large differences in fineness, especially if a slowly acting acid solution is used for the test.

Rubbings of white golds containing platinum group metals are attacked with far greater difficulty than those of alloys bleached with nickel or nickel and zinc. The development of an easily recognizable brown colouration of the acid drop during attack reveals the presence of palladium which is a constituent of many white golds.

\section{Influence of Other Alloy Constituents}

The effect of silver content on touching gold has been mentioned earlier in this article.

A clear indication that an unknown alloy contains additions to gold other than silver and copper is the impossibility of matching its colour with that of any needle in a comprehensive assortment of gold-silvercopper standards. If this is the case, the result of touching should be regarded as unreliable.

The presence of zinc and/or cadmium accelerates the rate of attack of rubbings by test acids, thereby resulting in different behaviour for the sample under test and a gold-silver-copper alloy needle of identical fineness. Consequently, the net result of touching alloys containing zinc and/or cadmium is usually an underestimation of their gold content. Platinum, on the other hand, has the opposite effect, even if present only in small quantities. Another effect of the addition of platinum to coloured golds is to impart to them a paler colour than that of platinum-free alloys.

\section{Touching Silver}

The presence of silver in an article of unknown composition is established by attacking a rubbing with dilute nitric acid, adding a drop of sodium chloride solution or a small crystal of sodium chloride and checking for the precipitation of silver chloride which dissolves in ammonia solution. Alternatively, the rubbing may be wetted with a solution of potassium dichromate and sulphuric acid, in which case silver forms its red-brown dichromate if present.

Usually, the fineness of silver alloys is determined simply by comparison of the colours of test rubbings with those of rubbings from needles of known composition. Test acids are employed only in special circumstances.
Touching is simplest on binary silver-copper alloys, owing to their wide range of colours - from red for a fineness of about 500 parts per thousand, pink around 600 , yellow between 700 and 800 and yellowish white to bright white for silver-rich alloys.

There is always an element of doubt concerning the results of touching silver, because this metal is seldom alloyed with copper alone. Additional metals, the presence of which cannot be deduced from the appearance of the sample, can have a significant effect on the colour of the rubbings. Thus, platinum-, palladium-, zinc- or cadmium-containing silver alloys produce rubbings of lighter colour than reference silver-copper binary alloys of identical fineness, which leads to an overestimation of the silver content of the former.

\section{Touching Platinum}

The presence of platinum is established by attacking or dissolving a rubbing with aqua regia while gently heating the touchstone, absorbing the aqua regia onto filter paper and adding a drop of zinc chloride solution to the damp spot on the paper. The development of a yellow to orange colour indicates platinum.

The determination to a reasonable accuracy of the fineness of platinum alloys by touching is restricted to those containing at least 900 parts per thousand of the metal. Alloys of lower fineness, which contain other precious metals and appreciable quantities of base metals, cannot be assayed for platinum by touching.

Platinum-rich alloys are tested on special, acidresistant stones. After preparation of the rubbings, the stone is immersed in a beaker with hot aqua regia to which potassium nitrate has been added. As soon as the attack is seen to begin, the stone is removed from the acid and placed under running water for observation.

There is no touchstone test for the other platinum group metals, including palladium. However, the latter can be detected qualitatively with a tin chloride solution, by the development on the touchstone of a brownish red colour which rapidly changes to dull greenish blue.

\section{Concluding Remarks}

This article has attempted to give the reader an insight into an important method, little known outside the jewellery industry, of analyzing for gold, silver and platinum (and sometimes other constituents) in alloys. The technique of touching has acceptable accuracy in most cases. Moreover, it is rapid and has the advantage of requiring only a very small amount of test alloy. It will undoubtedly retain its value as a method for the testing of precious metals and, in particular, gold alloys. 